\title{
Multi-modal Pipeline for Comprehensive Validation of Mitral Valve Geometry and Functional Computational Models
}

\author{
Dominik Neumann ${ }^{1,2}$, Sasa Grbic ${ }^{1,3}$, Tommaso Mansi $^{1}$, Ingmar Voigt ${ }^{1}$, \\ Jean-Pierre Rabbah ${ }^{4}$, Andrew W. Siefert ${ }^{4}$, Neelakantan Saikrishnan ${ }^{4}$, \\ Ajit P. Yoganathan ${ }^{4}$, David D. Yuh ${ }^{5}$, and Razvan Ionasec ${ }^{1}$ \\ 1 Imaging and Computer Vision, Siemens Corporate Technology, Princeton, NJ \\ 2 Pattern Recognition Lab, University of Erlangen-Nuremberg, Germany \\ 3 Computer Aided Medical Procedures, Technical University Munich, Germany \\ 4 The Wallace H. Coulter Department of Biomedical Engineering, Georgia Institute \\ of Technology and Emory University, Atlanta, GA \\ 5 Section of Cardiac Surgery, Department of Surgery, Yale University School of \\ Medicine, New Haven, CT
}

\begin{abstract}
Valvular heart disease affects a high number of patients, exhibiting significant mortality and morbidity rates. Mitral Valve (MV) Regurgitation, a disorder in which the MV does not close properly during systole, is among its most common forms. Traditionally, it has been treated with MV replacement. However, recently there is an increased interest in MV repair procedures, providing better long-term survival, better preservation of heart function, lower risk of complications, and usually eliminating the need for long-term use of blood thinners (anticoagulants). These procedures are complex and require an experienced surgeon and elaborate pre-operative planning. Hence, there is a need for efficient tools for training and planning of MV repair interventions. Computational models of valve function have been developed for these purposes. Nevertheless, state-of-the-art models remain approximations of real anatomy with considerable simplifications, since current modalities are limited by image quality. Hence, there is an important need to validate such low-fidelity models against comprehensive ex-vivo data to assess their clinical applicability. As a first step towards this aim, we propose an integrated pipeline for the validation of MV geometry and function models estimated in ex-vivo TEE data with respect to ex-vivo microCT data. We utilize a controlled experimental setup for ex-vivo imaging and employ robust machine learning and optimization techniques to extract reproducible geometrical models from both modalities. Using one exemplary case, we demonstrate the validity of our framework.
\end{abstract}

\section{Introduction}

The mitral valve (MV) separates the left atrium from the left ventricle and prevents the blood flow back to the left atrium during systole. Incorrect MV closure 
appears in many cardiac diseases and often requires MV replacement or repair surgery. In recent years, MV repair procedures, where the valve is surgically altered in order to restore its proper hemodynamic function, are substituting classical valve replacements [1]. It is the best option for nearly all patients with a regurgitant MV and for many with a narrowed (stenotic) MV [2]. However, the procedure is technically challenging and requires an experienced surgical team to achieve optimal results [1], since the deformation of complex valve anatomy during the intervention has to be predicted and associated with post-operative implications regarding valve anatomy and function. Having a framework to explore different surgical repair strategies for an individual patient and virtually compute their outcomes would be a desired tool in current clinical practice.

Driven by the growing prevalence of MV diseases, researchers are developing methods to assess MV anatomy from multiple imaging modalities and simulate its physiology using biomechanical models [3,4]. However, the clinical applicability is limited as they either do not enable patient-specific personalization of the geometric model or this process requires tedious manual interactions.

In recent years, methods have been proposed to delineate the MV using semi-manual or advanced automated algorithms [5]. Using these models, biomechanical computations can be performed based on a personalized patient-specific geometry as in [6]. However, their model relies on a simplified geometrical model, mainly due to the limitations of in-vivo TEE imaging. In particular, the MV leaflet clefts were not captured and the complex chordae anatomy was simplified with a parachute model. In order to apply such methods in clinical practice, the first step is to validate the prediction power of simplified models against ideal, high-fidelity models in a controlled ex-vivo environment.

We propose a novel validation framework for both geometric and biomechanical models extracted from non-invasive modalities. We developed a controlled experimental setup for MV ex-vivo imaging in order to acquire functional TEE data and high-resolution microCT images of the MV. Robust machine learning and optimization algorithms are utilized to produce accurate and reproducible models of the MV from both modalities. Based on the TEE model extracted during end-diastole (MV open), we utilize state of the art computational models to compute the geometric configuration of the MV during systole. We illustrate the capabilities of our framework using one excised sheep MV, showing promising results towards an integrated platform for comprehensive model validation.

\section{Experimental Setup}

In-Vitro Simulator. A novel closed-loop left heart simulator (Fig. 1) was utilized to carry out controlled in-vitro MV experiments [7,8]. It allows for precise control of annular and subvalvular MV geometry at physiological left heart hemodynamics. The modular design was optimized to allow for micro-computed tomography (microCT) and echocardiography techniques. The rigid left atrium and ventricle consists of a thin-walled acrylic chamber with a cylindrical cross section, resulting in uniform scattering and X-ray absorption for the microCT 


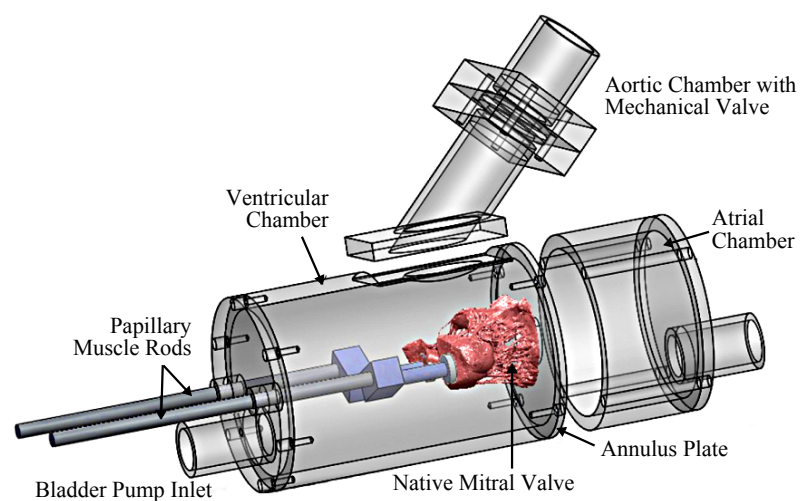

Fig. 1. Schematic representation of the in-vitro simulator with mounted mitral valve

and thus facilitating segmentation. The aortic chamber was designed at a $135^{\circ}$ angle from the MV to preserve their physiologic spatial orientation.

Experimental Protocol. For experimentation, a fresh ovine heart was obtained and the MV excised preserving its annular and subvalvular anatomy. All leaflet chordae of the selected MV are inserting directly into the papillary muscles (PM). The MV was sutured to the simulator's annulus using a Ford interlocking stitch. During valve suturing, care was taken to place each suture just above the valve's natural hinge and not through the leaflet tissue.

After annular suturing, each PM was attached to the PM control rods. Each PM was carefully positioned and fine-tuned to establish the control MV geometry as previously described [9]. The simulator was filled with $0.9 \%$ saline solution and leaflet dynamics and coaptation geometry were studied at room temperature under physiologic hemodynamic conditions $(120 \mathrm{mmHg}$ peak left ventricular pressure, $5.0 \mathrm{~L} / \mathrm{min}$ average cardiac output at a heart rate of $70 \mathrm{bpm}$ ) to ensure proper valvular function. 3D echocardiography at a good temporal resolution of $50 \mathrm{~Hz}$ was acquired using a Phillips iE33 system with an X7-2 pediatric probe.

MicroCT Protocol. The atrial chamber and aortic section were removed from the left heart simulator, and the left ventricle was fixed to the microCT gantry using a custom adaptor plate. The MV geometry did not get perturbed by these changes. The valve was scanned in air under $\approx 30 \mathrm{mmHg}$ ventricular pressure using a viva CT 40 system (Scanco Medical AG). The geometry was acquired at $39 \mu \mathrm{m}$ voxel size $(\approx 600$ slices $)$ using scanning parameters optimized for low density soft tissues ( $55 \mathrm{keV}$ energy, $109 \mathrm{uA}$ intensity, $300 \mathrm{~ms}$ integration time).

\section{Methods}

\subsection{Extraction of High-Fidelity Model from Ex-Vivo MicroCT}

We propose a novel automated segmentation procedure for microCT images of the MV (see Fig. 2), where the structure of the valve is decomposed into parts relevant to the subsequent computation of geometric measurements. 


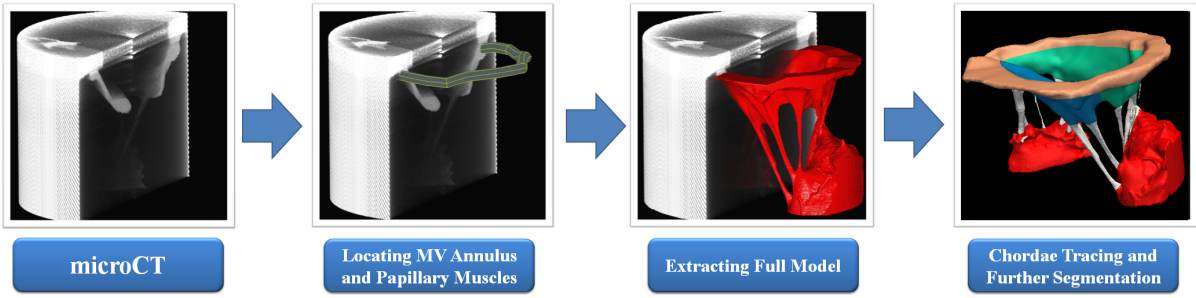

Fig. 2. Left: microCT scan of MV suspended in the simulator, right: extracted model

Locating MV Annulus and Papillary Muscles. An adaption of the approach proposed in [10] based on convex programming is exploited for the localization task. Efficient implementations as described in [11] allow for exhaustive parameter search within seconds. We utilize a sparse model of the MV, which can be generated manually or extracted automatically from corresponding TEE models. Instead of directly using the raw image, a smooth Euclidean distance transform of the MV is required. We approximate the transform by a distance map based on an anisotropically filtered image derived from the microCT scan using Danielsson's method [12]. The described workflow allows for the extraction of rough estimates of the centers of the papillary muscles, several points on the mitral annulus, and points in the posterior and anterior leaflet.

Extracting Full Model. Based on the estimated locations of the mitral annulus and papillary muscles, we generate seed points for the Random Walker algorithm, yielding a segmentation of the MV in the original image. The parameters were set heuristically. The resulting mask is then converted into a triangulated mesh model for the remaining model decomposition steps utilizing the Marching Cube algorithm.

Chordae Tracing. Given the MV mesh and points in each papillary muscle (PM) and on the annulus, we estimate the configuration of the chordae tendineae, which we use to determine the exact locations of the chordae insertion points, i.e. those locations, where a chord is connected to the leaflet. This information is necessary in order to split the MV mesh into PMs, chordae, annulus and leaflets. Chordae segmentation is not straightforward, since the chordae are structured in a tree-like fashion and a chord is not necessarily a straight line, it rather shows high curvature under certain conditions. Hence, we perform a novel path tracing approach based on geodesic distances on the mesh, where we determine all paths from the annulus to the PMs (start and end point are given by the sparse model estimation). It is a valid assumption that each of those paths contains a chord. Starting from the annulus, for each point on the descending path, we compute the mesh thickness at this location and retrieve the leaflet insertion points by analyzing the thickness profile throughout the path. A threshold of lower than $0.5 \mathrm{~mm}$ thickness on the descending path from the annulus was determined to be the location of an insertion point. Next, we cut the mesh at the insertion point and repeat the previous procedure until no more paths connecting the mitral annulus with a one of the papillary muscles are found. 

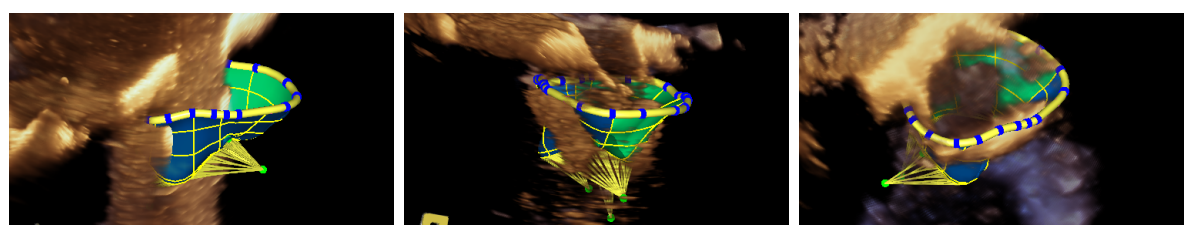

Fig. 3. Estimated MV model from ex-vivo TEE scan visualized in the TEE volume

\subsection{TEE Mitral Valve Apparatus Parametrization and Estimation}

In this study, we utilize the non-invasive anatomical point distribution model $\mathcal{S}$ of the MV and its subvalvular components from $[5,13]$ estimated on 3D TEE. $\mathcal{S}$ comprises mitral annulus, and anterior and posterior leaflets and papillary tips. Nine anatomical landmarks (two trigones, two commissures, one posterior annulus mid-point, two leaflet tips, and two papillary tips) allow for a consistent, patient-specific derivation of $\mathcal{S}$, which is capable of capturing a broad spectrum of morphological variations. The parameters of $\mathcal{S}$ are incrementally estimated within the hierarchical, discriminative Marginal Space Learning framework using classifiers based on the Probabilistic Boosting Tree with Haar-like and Steerable features. Fig. 3 depicts the extracted model based on the TEE image.

\subsection{Biomechanical Model of the Mitral Valve}

MV closure is calculated from the TEE anatomy based on the model proposed in [6]. In brief, the dynamics system $\mathrm{M} \ddot{\boldsymbol{u}}+\mathrm{C} \boldsymbol{u}+\mathrm{K} \boldsymbol{u}=\boldsymbol{f}_{t}+\boldsymbol{f}_{p}+\boldsymbol{f}_{c}$ is solved, where $\mathrm{M}$ is the diagonal mass matrix calculated from the mass density $\rho=1040 \mathrm{~g} / \mathrm{L}, \mathrm{C}$ is the Rayleigh damping matrix with coefficients $1 e 4 s^{-1}$ and $0.1 s$ for the mass and stiffness matrix respectively, $\mathrm{K}$ is the stiffness matrix, $\boldsymbol{f}_{t}$ is the force created by the chords on the leaflets, $\boldsymbol{f}_{p}$ the pressure force, $\boldsymbol{f}_{c}$ the contact forces and $\boldsymbol{u}$ the displacement. In this study, we rely on transverse isotropic linear tissue elasticity implemented using a co-rotational finite elements method (FEM) to cope with large deformations. The choice of linear elasticity is motivated by recent findings suggesting a linear behavior in-vivo [14]. Poisson ratio is $\nu=0.488$ for both leaflets, fiber Young's modulus is $E_{A L}=6.23 \mathrm{MPa}$ and $E_{P L}=2.09 \mathrm{MPa}$ for the anterior and posterior leaflets respectively, cross-fiber Young's modulus is $E_{A L}=2.35 \mathrm{MPa}$ and $E_{P L}=1.88 \mathrm{MPa}$ and shear modulus is $1.37 \mathrm{MPa}$. Mitral annulus and PMs are fixed. Chordae are modeled as in [6]: twenty-eight marginal chordae are evenly attached at the free-edges of the leaflets and four chordae are tethered at the base of the leaflets. In that way, we mimic a real-case scenario where the detailed configuration of the chordae is unknown. Chordae follow an exponential law [6]. Finally, self collisions are modeled, with collision stiffness of $100 \mathrm{kPa}$ and friction coefficient of 0.1 . The model is implemented in the SOFA framework ${ }^{6}$. Spatial variables are discretized using linear tetrahedra while an Euler implicit time discretization is employed for robust computation.

\footnotetext{
${ }^{6}$ http://www.sofa-framework.org/
} 

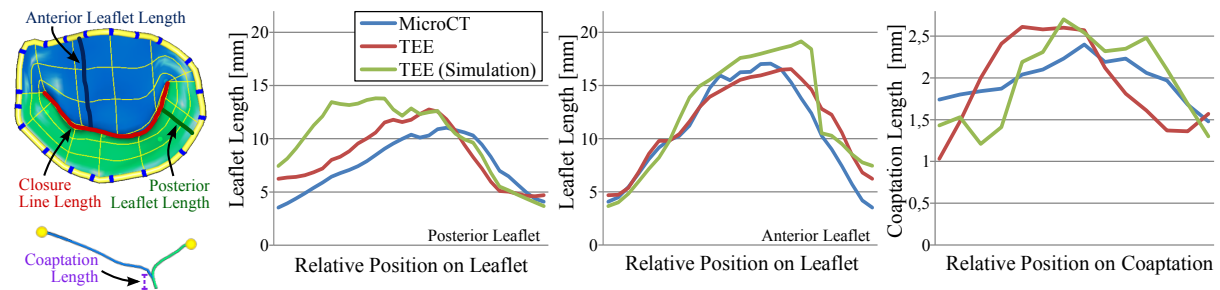

Fig. 4. Geometric comparison at systole from microCT, annotated and simulated TEE

\section{Experimental Results}

We utilize our framework on one ovine valve and compare the geometric configuration between the model constructed from TEE and microCT during systole (MV closed). In addition, we compute the MV geometry at systole from an enddiastolic (MV open) TEE image and compare it to the ground-truth geometrical configuration obtained from the microCT image.

\subsection{Geometric Comparison}

Based on the geometric models extracted during systole (MV closed) from TEE and microCT, we measured clinically relevant parameters (coaptation length, coaptation area, closure line length, and anterior and posterior leaflet length, see Fig. 4, left panel) in order to quantitatively compare geometric differences between the two models (see Fig. 4, right panels). The maximum coaptation length is $2.41 \mathrm{~mm}$ vs $2.62 \mathrm{~mm}$ for microCT and TEE, respectively. Closure line lengths are measured as $23.08 \mathrm{~mm}$ vs $21.1 \mathrm{~mm}$, and coaptation areas are $45.8 \mathrm{~mm}^{2}$ vs $41.1 \mathrm{~mm}^{2}$. Qualitative comparisons are shown in Fig. 5. These results indicate that the utilized simplified TEE model can accurately represent important biomarkers compared to an idealized model (extracted from microCT).

\subsection{Closure Computation}

Starting from the end-diastolic TEE MV model (last frame where the MV is seen open in the TEE image), we computed MV closure based on the model described in Sec. 3.3. In this experiment, we used a time step of $10 \mathrm{~ms}$. A nominal pressure profile varying from $0 \mathrm{mmHg}$ to $120 \mathrm{mmHg}$ was applied [6]. Anterior leaflet chord rest length was set to $1.6 \times$ longer than the distance between free-edges and papillary muscles to cope with folded chords at end-diastole (MV open). That ratio was estimated on other microCT data. Finally, to capture the fast dynamics and correctly account for collisions and inertia, pressure increase duration was scaled to last $10 \mathrm{~s} .1000$ iterations were calculated.

Fig. 6 illustrates the calculated MV closure geometry from time $0 \mathrm{~s}$ to $7 \mathrm{~s}$, before peak pressure was reached (afterwards the valve stayed closed). As one can see, our model could capture MV closure qualitatively well. Quantitatively, 

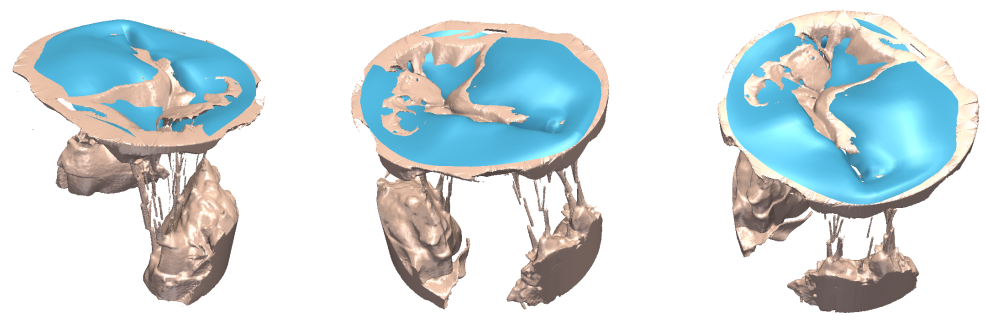

Fig. 5. Qualitative comparison of MV geometry from TEE (cyan) and microCT (beige)

we measured clinically-related parameters as described above from the predicted closure and compared them against the idealized model (Fig. 4). Maximum coaptation length is $2.71 \mathrm{~mm}$ for the computed model vs $2.41 \mathrm{~mm}$ for the idealized microCT model. Closure line lengths are measured as $23.3 \mathrm{~mm}$ vs $23.08 \mathrm{~mm}$, and coaptation areas are $46.1 \mathrm{~mm}^{2}$ vs $45.8 \mathrm{~mm}^{2}$. These results confirm that simplified models from TEE can be utilized to build biomechanical models and compute accurate MV closure geometry in respect to relevant clinical parameters.

\section{Conclusion}

We proposed a novel complete pipeline for validating geometrical and functional models of the mitral valve utilizing a controlled ex-vivo setup capable of acquiring both high-resolution ex-vivo microCT scans in order to obtain ground-truth information, and standard low-resolution TEE images. This pipeline serves as a bridge between ex-vivo and non-invasive clinical modalities. We integrated robust algorithms in order to extract reproducible models from microCT and TEE images. Thus, quantitative comparison of clinically relevant measurements is possible. Measurements were computed from simplified models extracted from non-invasive modalities and compared to idealized high-fidelity models based on microCT. Finally, experiments on real data obtained from one ovine valve were conducted as a proof of concept, demonstrating the capabilities of our framework. Now that we have an integrated and comprehensive ex-vivo microCT / TEE setup, we are able to evaluate models in a consistent manner on a larger
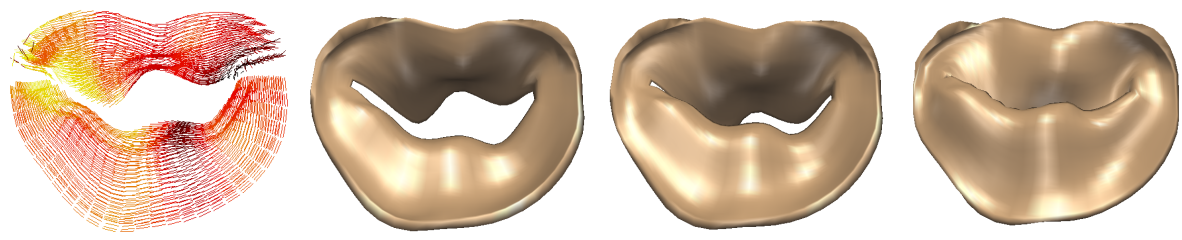

Fig. 6. Left: fiber orientations of computational TEE model, right: different time steps of computed MV closure geometry, initialized from end-diastolic (MV open) TEE image 
population and thus validate the prediction power of current in-vivo computational frameworks, which will be the next step of the current study.

\section{References}

1. Kilic, A., Shah, A., Conte, J., Baumgartner, W., Yuh, D.: Operative outcomes in mitral valve surgery: Combined effect of surgeon and hospital volume in a population-based analysis. J. Thorac. Cardiovasc. Surg. (2012)

2. Bolling, S., Li, S., O’Brien, S., Brennan, M., Prager, R., Gammie, J.: Predictors of mitral valve repair: Clinical and surgeon factors. Ann. of Thorac. Surg. 90 (2013) 1904-1912

3. Wang, Q., Sun, W.: Finite element modeling of mitral valve dynamic deformation using patient-specific multi-slices computed tomography scans. Ann. Biomed. Eng. 41(1) (2013) 142-153

4. Stevanella, M., Maffessanti, F., Conti, C., Votta, E., Arnoldi, A., Lombardi, M., Parodi, O., Caiani, E., Redaelli, A.: Mitral valve patient-specific finite element modeling from cardiac mri: Application to an annuloplasty procedure. Cardiovascular Engineering and Technology 2(2) (2011) 66-76

5. Ionasec, R., Voigt, I., Georgescu, B., Wang, Y., Houle, H., Vega-Higuera, F., Nassir, N., Comaniciu, D.: Patient-specific modeling and quantification of the aortic and mitral valves from 4-D cardiac CT and tee. TMI 29(9) (2010) 1636-1651

6. Mansi, T., Voigt, I., Georgescu, B., Zheng, X., Assoumou Mengue, E., Hackl, M., Ionasec, Razvan, T., Seeburger, J., Comaniciu, D.: An integrated framework for fnite-element modeling of mitral valve biomechanics from medical images: Application to mitralclip intervention planning. Med. Image Anal. 16(7) (2012) 1330-1346

7. Siefert, A.W., Rabbah, J.P.M., Koomalsingh, K.J., Touchton Jr, S.A., Saikrishnan, N., McGarvey, J.R., Gorman, R.C., Gorman III, J.H., Yoganathan, A.P.: In vitro mitral valve simulator mimics systolic valvular function of chronic ischemic mitral regurgitation ovine model. Ann. Thorac. Surg. 95 (2013) 825-830

8. Rabbah, J.P., Saikrishnan, N., Yoganathan, A.P.: A novel left heart simulator for the multi-modality characterization of native mitral valve geometry and fluid mechanics. Ann. Biomed. Eng. 31 (2012) 305-315

9. Jimenez, J.H., Soerensen, D.D., He, Z., He, S., Yoganathan, A.P.: Effects of a saddle shaped annulus on mitral valve function and chordal force distribution: an in vitro study. Ann. Biomed. Eng. 31(10) (2003) 1171-1181

10. Chen, S.S., Donoho, D.L., Saunders, M.A.: Atomic decomposition by basis pursuit. SIAM 43(1) (2001) 129-159

11. Breitenreicher, D., Schnoerr, C.: Model-based multiple rigid object detection and registration in unstructured range data. International Journal of Computer Vision 92(1) (2011) 1573-1405

12. Danielsson, P.: Euclidean distance mapping. Computer Graphics and Image Processing 14(3) (1980) 227-248

13. Voigt, I., Mansi, T., Ionasec, R., Assoumou Mengue, E., Houle, H., Georgescu, B., Hornegger, J., Comaniciu, D.: Robust physically-constrained modeling of the mitral valve and subvalvular apparatus. In: MICCAI. Volume 6893 of LNCS. Springer Berlin Heidelberg (2011) 504-511

14. Krishnamurthy, G., Itoh, A., Bothe, W., Swanson, J., Kuhl, E., Karlsson, M., Craig Miller, D., Ingels, N.: Stress-strain behavior of mitral valve leaflets in the beating ovine heart. J. Biomech. 42(12) (2009) 1909-1916 